\title{
The place and role of rehabilitation in patients undergoing endovascular revascularization for chronic critical ischemia
}

\author{
Bogdan Duţu', Dana Pop ${ }^{1,2}$, Dumitru Zdrenghea ${ }^{1,2}$, Horatiu Comsa ${ }^{1,2}$, Adrian Corneliu Iancu ${ }^{2,3}$
}

Corresponding author: Dana Pop, E-mail address: pop67dana@gmail.com

\author{
${ }^{1}$ Clinical Rehabilitation Hospital Cluj-Napoca, Romania \\ 2 "Iuliu Haţieganu” University of Medicine and Pharmacy Cluj-Napoca, Romania \\ 3 “Niculae Stancioiu” Heart Institute Cluj-Napoca, Romania
}

\begin{abstract}
The monitoring algorithm after endovascular revascularization in critical limb ischemia (CLI) involves the preservation of patent circulation in the affected limb and patient care optimization. A protection of both revascularized arterial and systemic circulation, especially through a reduction of atherosclerotic load, is required. The majority of the patients with CLI are elderly and have multiple comorbidities. In this context, a long-term approach to critical ischemia and the maintenance of limb mobility can contribute to an improvement in the quality of life and overall prognosis of the patients. This involves a change of lifestyle, the control of cardiovascular risk factors and the correct application of secondary prevention measures (medication and rehabilitation).
\end{abstract}

Key words: critical lower limb ischemia, revascularization, lifestyle change, rehabilitation

\section{Introduction.}

Critical limb ischemia (CLI) is a severe, frequently disabling disorder (risk of amputation, limb revascularization/salvage surgery, with increased morbidity and mortality, with considerable consequences on prognosis). According to the Fontaine classification, critical ischemia is characteristic of advanced stages (III and IV) of peripheral arterial occlusive disease, being a disorder in which the integrity of the affected limb is threatened, but which may also involve a high vital risk. The Trans-Atlantic Consensus II reported that $30 \%$ of patients with CLI require amputation and that $25 \%$ of patients with amputations die during the next year of follow-up, which confirms the extremely poor prognosis of CLI [1]. Atherosclerosis represents the basis of the development and progression of arterial occlusive disease. Over the past period (the last two decades), due to the progress of medicine and minimally invasive techniques, endovascular revascularization therapy has been the first line method used for the treatment of this disease. At the same time, a change in lifestyle, adequate drug therapy and physical exercise are mandatory over the long term in this category of patients.

The main objectives after revascularization are the use of optimal drug therapy, periodic evaluation of the repermeabilized arterial bed, reintervention depending on the case (endovascular or surgical), and long-term rehabilitation.

\section{Lifestyle changing}

Smoking is the most important risk factor in peripheral arterial disease and implicitly in critical ischemia. The duration of exposure to smoking is correlated with the severity of the disease, the percentage of lower limb amputations, as well as mortality [2]. Quitting smoking is the most effective strategy regarding the cost/benefit ratio in the long term [3]. The efficiency of nicotine replacement therapy was assessed in a Cochrane analysis of 132 randomized trials [4]. In these, all available nicotine replacement forms (chewing gum, nasal spray, inhaler, sublingual tablet, transdermal patch) were used, which led to a 50-70\% interruption of smoking [4]. A number of randomized trials monitored the use of bupropion, an antidepressive drug, in smokers with cardiovascular disease. Abstinence rates of 34\%, 27\% and 22\% were reported at 3, 6 and 12 months of follow-up compared to $15 \%, 11 \%$ and $9 \%$ with placebo [5]. Furthermore, varenicline proved to be superior to bupropion, while the important respiratory benefits secondary to smoking cessation should also be mentioned [6].

\section{Dyslipidemia.}

High total cholesterol and especially high LDL, increased circulating levels of triglycerides and lipoprotein are major independent risk factors for peripheral arterial disease. In patients with critical ischemia, statins are the first line agents used in the fight against and control of atherogenic dyslipidemia, 
in the maximum dose tolerated by the patient [3]. It is recommended to reach an LDL level lower than 70 $\mathrm{mg} / \mathrm{dl}$ [7]. Statins also have a well established role in the secondary prevention of cardiovascular events [3].

\section{Diet}

A diet rich in fruits and vegetables, poor in unsaturated fatty acids [3], low in carbohydrates in the case of diabetes mellitus and low in sodium in hypertensive patients is recommended. At the same time, this can ensure a $\mathrm{BMI}<25 \mathrm{~kg} / \mathrm{m}^{2}$ [3].

\section{Secondary drug prevention.}

Antiplatelet treatment (aspirin or clopidogrel) is indicated in patients with critical ischemia, their association being required in those undergoing percutaneous angioplasty [7].

Vasoactive medication, represented by prostaglandins, is the only medication with demonstrated efficiency in patients not eligible for revascularization or those in whom these invasive methods (endovascular or surgical) have failed [2].

\section{Physical training and lower limb rehabilitation. Physical activity}

There is strong evidence supporting the fact that physical training is beneficial in patients suffering from claudication (arterial occlusive disease stage II Fontaine), especially if physical activity is supervised [8]. The European Society of Cardiology Guidelines mention that most of the studies were based on supervised exercise programs over a period of at least 3 months, with a frequency of at least three times a week [7]. The American guidelines on peripheral arterial disease recommend for intermittent claudication a rehabilitation program based on physical training for 30-45 minutes daily, at least 3 times a week, over a period of 12 months [9]. A metaanalysis published in 1995 showed that almost maximal physical exercise led to a significant improvement in the walking distance [10]. Another analysis based on 30 studies demonstrated that a 2year exercise program resulted in a mean decrease of the pain threshold by 82 meters and an increase of the walking distance by approximately 109 meters [11]. Furthermore, a Cochrane analysis of 22 clinical trials including 1200 patients with peripheral arterial disease who attended rehabilitation programs demonstrated a significant improvement in the walking distance in patients with physical training compared to classical training or placebo [12]. Unsupervised physical exercise is not as effective as rehabilitation programs supervised by professionals. At the same time, these are a beneficial alternative with positive overall effects on quality of life. Physical exercise is not feasible in patients with critical ischemia, but can be taken into consideration after revascularization procedures [7].

The potential value of supervised physical training in patients with critical ischemia undergoing percutaneous revascularization has not been studied. This could be explained by the fact that these patients are, on the one hand, fragile, and on the other hand, multiple comorbidities are associated which might compromise an exercise program or make it impossible to put into practice [13]. However, once having reached the critical ischemia stage, patients suffer from pain, they have wounds or amputations and subsequently, their mobilization ability will decrease, which results in a significant reduction of physical activity. So far there are no studies demonstrating the benefits of activity in critical ischemia and implicitly, its effect on quality of life remains unclear.

Nonetheless, physical training might be beneficial in patients with CLI who become asymptomatic at rest or those with post-revascularization exertional claudication. Peripheral arterial disease guidelines recommend physical training for all patients after revascularization, as well as the express inclusion of patients with amputations in supervised rehabilitation programs [7]. It should not be forgotten that physical activity plays an important role in the prevention and progression of atherosclerosis.

\section{Treatment of associated disorders}

Arterial hypertension is a major risk factor for peripheral arterial disease. Current guidelines indicate aggressive treatment in absolute value for hypertension in patients with arterial occlusive disease [7]. In critical ischemia, normally high blood pressure values are recommended, so as not to affect distal perfusion pressure in the affected limb. It is recommended to use angiotensin converting enzyme inhibitors with an established benefit for Ramipril, according to the HOPE study conducted in more than 4000 patients [14]. Beta-blockers are especially used 
in patients undergoing revascularization surgery, for cardiovascular protection, not being contraindicated in other situations [2].

Diabetes mellitus is a major risk factor for cardiovascular disease in general, contributing to the negative prognosis of its complications (heart failure) [3,15]. Advanced age, male sex, smoking, arterial hypertension and diabetes mellitus are the most important risk factors in the development of peripheral arterial disease [3].

A high proportion of patients with critical limb ischemia (CLI) are diabetic and difficult to treat. The results of endovascular and surgical treatment are supposed to be similar, but given that diabetic patients are in an advanced stage of peripheral arterial occlusive disease, they have low success rates of primary patency after revascularization [13]. However, through an early and adequate use of salvage reinterventions, acceptable rates of secondary permeability and limb salvage can be obtained [13]. This is why diabetic patients represent a subgroup that should be closely watched during clinical evolution monitoring. HbA1c must be maintained below $7 \%$ through an adequate diet and hypoglycemic medication [3].

At the same time, patients with CLI have an increased prevalence of ischemic heart disease, which significantly increases the risk of cardiac mortality and morbidity. Thus, all patients should be considered as having a high risk for clinically significant ischemic heart disease and should be assessed accordingly [3,7]. Patients with severe ischemic symptoms require coronarography and interventional treatment. Beta-blockers, aspirin, statins and angiotensin converting enzyme inhibitors or sartans should be included in the therapeutic plan of these patients [3].

The proportion of patients with critical ischemia and concomitant chronic renal failure is increasing. With an aggressive invasive approach through repeated revascularizations, arterial patency rates come close to those of patients with normal renal function. The risk of amputation still remains high in these patients [13].

Elderly. After the age of 80 years, the most important predictor of the mobilization capacity is the patient's general status at presentation, which includes mental status and the presence or absence of self-care. Usually, octogenarian patients with critical ischemia who preserve their pre-interventional mobility have a favorable evolution even in the case of infrageniculate arterial reconstructions [16]. At one year, $88 \%$ of these can mobilize independently, $85 \%$ live within their home perimeter, and $80 \%$ fit into both situations, while after 5 years, $71 \%$ maintain their mobility and $81 \%$ live independently [17].

\section{Conclusion}

The follow-up and continuous monitoring of patients with endovascular revascularization for critical limb ischemia is mandatory in order to ensure the best survival, limb salvage and stabilization outcomes. These objectives are achieved by adequate drug therapy, careful monitoring of the patency of the vascular axis responsible for the perfusion and viability of the threatened limb, and not least, by changing lifestyle and applying secondary preventive measures.

\section{Conflict of interest/financing}

None

\section{Bibliography}

1. Norgren L, Hiatt WR, Dormandy JA, Nehler MR, Harris KA, Fowkes FG; TASC II Working Group. Inter-Society Consensus for the Management of Peripheral Arterial Disease (TASC II). J Vasc Surg. 2007;45 Suppl S:S5-67.

2. Diehm N, Schmidli J, Setacci C, Ricco JB, de Donato G, Becker F, et al. Management of Cardiovascular Risk Factors and Medical Therapy. Eur J Vasc Endovasc Surg. 2011;42 Suppl 2:S33-42.

3. Piepoli MF, Hoes AW, Agewall S, Albus C, Brotons C, Catapano AL, et al. Authors/Task Force Members. 2016 European Guidelines on cardiovascular disease prevention in clinical practice: The Sixth Joint Task Force of the European Society of Cardiology and Other Societies on Cardiovascular Disease Prevention in Clinical Practice (constituted by representatives of 10 societies and by invited experts). Developed with the special contribution of the European Association for Cardiovascular Prevention \& Rehabilitation (EACPR). Eur. Heart J. 2016;37:2315-2381.

4. Stead LF, Perera R, Bullen C, Mant D, HartmannBoyce J, Cahill K, Lancaster T. Nicotine replacement therapy for smoking cessation. 2012;11:CD000146.

5. Tonstad S, Farsang C, Klaene G, Lewis K, Manolis A, Perruchoud AP, et al. Bupropion SR for smoking cessation in smokers with cardiovascular disease: a multicentre, randomised study. Eur Heart J 2003;24(10):946-55. 
6. Cahill K, Stead LF, Lancaster T. Nicotine receptor partial agonists for smoking cessation. Cochrane Database Syst Rev. 2012; 2:CD006103.

7. Aboyans V, Ricco JB, Bartelink ML, Björck M, Brodmann M, Cohnert T, et al. 2017 ESC Guidelines on the Diagnosis and Treatment of Peripheral Arterial Diseases, in collaboration with the European Society for Vascular Surgery (ESVS). Eur J Vasc Endovasc Surg. 2017; pii: S1078-5884(17)30454-9.

8. McDermott MM. Exercise Rehabilitation for Peripheral Artery Disease: A review. J Cardiopulm Rehabil Prev. 2018;38(2):63-69.

9. Gerhard-Herman MD, Gornik HL, Barrett C, Barshes NR, Corriere MA, Drachman DE, et al. 2016 AHA/ACC Guideline on the Management of Patients With Lower Extremity Peripheral Artery Disease: A Report of the American College of Cardiology/American Heart Association Task Force on Clinical Practice Guidelines. Circulation. 2017;135(12):e726-e779.

10. Gardner AW, Poehlman ET. Exercise rehabilitation programs for the treatment of claudication pain. A meta-analysis. JAMA 1995;274:975-980.

11. Lane R, Ellis B, Watson L, Leng GC. Exercise for intermittent claudication. Cochrane Database Syst Rev 2014;7:CD000990.

12. Watson L, Ellis B, Leng GC. Exercise for intermittent claudication. Cochrane Database Syst Rev 2008;4:CD000990.

13. F. Dick, J.-B. Ricco, A.H. Davies, P. Cao, C. Setacci, G. de Donato, et al. Chapter VI: Follow-up after Revascularisation. Eur J Vasc Endovasc Surg. 2011;42 Suppl 2:S75-90.

14. Heart Outcomes Prevention Evaluation Study Investigators, Yusuf S, Sleight P, Pogue J, Bosch J, Davies R, Dagenais G. Effects of an angiotensinconverting-enzyme inhibitor, ramipril, on cardiovascular events in high-risk patients. N Engl J Med. 2000;342(3):145-53.

15. Sitar Taut AV, Pop D, Zdrenghea DT. NT-proBNP values in elderly heart failure patients with atrial fibrillation and diabetes. J Diabetes Complications. 2015;29(8):1119-23.

16. Ballotta E, Gruppo M, Mazzalai F, Martella B, Terranova O, Da Giau G. Infrapopliteal arterial reconstructions for limb salvage in patients aged $>$ or $=$ 80 years according to preoperative ambulatory function and residential status. Surgery 2010;148:119-28.

17. Taylor SM, Kalbaugh CA, Blackhurst DW, Cass AL, Trent EA, Langan 3rd EM, Youkey JR. Determinants of functional outcome after revascularization for critical limb ischemia: An analysis of 1000 consecutive vascular interventions. J Vasc Surg 2006;44:747-55. 